Proceedings of the Edinburgh Mathematical Society (2004) 47, 679-694 (C)

DOI:10.1017/S0013091502001165 Printed in the United Kingdom

\title{
OPERATORS THAT ARE NUCLEAR WHENEVER THEY ARE NUCLEAR FOR A LARGER RANGE SPACE
}

\author{
EVE OJA \\ Faculty of Mathematics and Computer Science, Tartu University, \\ Liivi 2, EE-50409 Tartu, Estonia (eveoja@math.ut.ee)
}

(Received 20 December 2002)

\begin{abstract}
Let $X$ be a Banach space and let $Y$ be a closed subspace of a Banach space $Z$. The following theorem is proved. Assume that $X^{*}$ or $Z^{*}$ has the approximation property. If there exists a bounded linear extension operator from $Y^{*}$ to $Z^{*}$, then any bounded linear operator $T: X \rightarrow Y$ is nuclear whenever $T$ is nuclear from $X$ to $Z$. The particular case of the theorem with $Z=Y^{* *}$ is due to Grothendieck and Oja and Reinov. Numerous applications are presented. For instance, it is shown that a bounded linear operator $T$ from an arbitrary Banach space $X$ to an $\mathcal{L}_{\infty}$-space $Y$ is nuclear whenever $T$ is nuclear from $X$ to some Banach space $Z$ containing $Y$ as a subspace.
\end{abstract}

Keywords: nuclear operators; approximation property; extension operator; projective tensor products

2000 Mathematics subject classification: Primary 46B20; 46B28; 47B10

\section{Introduction}

Let $X$ and $Y$ be Banach spaces. A bounded linear operator $T \in \mathcal{L}(X, Y)$ is said to be nuclear if there exist $x_{n}^{*} \in X^{*}$ and $y_{n} \in Y$ such that $\sum_{n=1}^{\infty}\left\|x_{n}^{*}\right\|\left\|y_{n}\right\|<\infty$ and $T x=\sum_{n=1}^{\infty} x_{n}^{*}(x) y_{n}$ for all $x \in X$. In this case, one writes $T=\sum_{n=1}^{\infty} x_{n}^{*} \otimes y_{n}$ and calls the latter sum a nuclear representation of $T$. Let us denote by $\mathcal{N}(X, Y)$ the collection of all nuclear operators from $X$ to $Y$.

Every operator $T \in \mathcal{L}(X, Y)$ may be viewed as an operator from $X$ to $Y^{* *}$ considering the operator $j_{Y} T$, where $j_{Y}: Y \rightarrow Y^{* *}$ denotes the canonical embedding. Grothendieck proved, in his famous memoir [7, Chapter I, pp. 85, 86], that

$$
j_{Y} T \in \mathcal{N}\left(X, Y^{* *}\right) \Rightarrow T \in \mathcal{N}(X, Y)
$$

whenever $X^{*}$ has the approximation property. He also affirmed (see [6, p. 17] and [7, Chapter I, p. 86]) that implication (1.1) holds whenever the second dual space $Y^{* *}$ has the approximation property. A counterexample to this affirmation of Grothendieck was given by $\mathrm{Oja}$ and Reinov (see $[\mathbf{2 4}]$ or $[\mathbf{2 5}]$ ). On the other hand, they proved [25] that implication (1.1) is true whenever the third dual space $Y^{* * *}$ has the approximation property. The latter assumption seems to be rather confusing because one does not know 
much about the third duals of non-reflexive Banach spaces, even of so-called classical ones (for instance, let us quote Diestel $[\mathbf{1}$, p. 35$]$ here: ' $b a=c_{0}^{* * *}$ is the best I can do').

The purpose of the present article is to extend the above-described Grothendieck-OjaReinov theorem to a more general natural setting in such a way that the assumption about the approximation property would solely concern the first dual spaces. In particular, this clarifies the appearance of the third dual $Y^{* * *}$ in the Oja-Reinov result.

To state the main result of this article, Theorem 1.1 below, we need the notion of an extension operator. Let $Y$ be a closed subspace of a Banach space $Z$. An operator $\Phi \in \mathcal{L}\left(Y^{*}, Z^{*}\right)$ is called an extension operator if $\left(\Phi y^{*}\right)(y)=y^{*}(y)$ for all $y^{*} \in Y^{*}$ and all $y \in Y$. Remark that the existence of an extension operator is equivalent to the annihilator of $Y$ being complemented in $Z^{*}$.

Let us also recall that the nuclear norm $\|T\|_{\mathcal{N}}$ of a nuclear operator $T \in \mathcal{N}(X, Y)$ is defined by the equality

$$
\|T\|_{\mathcal{N}}=\inf \left\{\sum_{n=1}^{\infty}\left\|x_{n}^{*}\right\|\left\|y_{n}\right\|: T=\sum_{n=1}^{\infty} x_{n}^{*} \otimes y_{n}\right\},
$$

where the infimum is taken over all possible nuclear representations of $T$. It is straightforward to verify that if there are two more bounded linear operators $A$, acting to $X$, and $B$, acting from $Y$, then $B T A$ is a nuclear operator and $\|B T A\|_{\mathcal{N}} \leqslant\|B\|\|T\|_{\mathcal{N}}\|A\|$.

Theorem 1.1. Let $X$ be a Banach space. Let $Y$ be a closed subspace of a Banach space $Z$ and let $j: Y \rightarrow Z$ denote the identity embedding. Assume that there is an extension operator $\Phi \in \mathcal{L}\left(Y^{*}, Z^{*}\right)$. If $X^{*}$ or $Z^{*}$ has the approximation property, then, for every operator $T \in \mathcal{L}(X, Y)$, the following implication holds:

$$
j T \in \mathcal{N}(X, Z) \Rightarrow T \in \mathcal{N}(X, Y) .
$$

Moreover,

$$
\frac{1}{\|\Phi\|}\|T\|_{\mathcal{N}} \leqslant\|j T\|_{\mathcal{N}} \leqslant\|T\|_{\mathcal{N}}
$$

Pairs of Banach spaces $Z$ and their closed subspaces $Y$ for which there exists an extension operator $\Phi \in \mathcal{L}\left(Y^{*}, Z^{*}\right)$ were systematically studied by Fakhoury $[\mathbf{3}]$ and Kalton [11]. The existence of $\Phi$ with $\|\Phi\|=1$ means, according to the terminology of Godefroy, Kalton and Saphar [5], that $Y$ is an ideal in $Z$. Different subclasses of ideals have been extensively studied by many authors (for references see $[\mathbf{2 2}, \S 4]$ ).

Theorem 1.1 obviously contains the Grothendieck-Oja-Reinov result as a special case when $Z=Y^{* *}$ and $\Phi=j_{Y^{*}}$. Other applications of Theorem 1.1 will be discussed in $\S 5$ of this article.

Sections 2 and 3 contain preliminaries and preparatory results needed for the proof of Theorem 1.1. For instance, we prove (see Proposition 2.1) that the approximation property of a Banach space $Z$ is inherited by its closed subspace $Y$ whenever there exists an extension operator $\Phi \in \mathcal{L}\left(Y^{*}, Z^{*}\right)$. This result is essentially due to Kalton [11] and Lima [12], but we give here a direct proof that does not rely on the principle of local reflexivity. We also prove (see Theorems 3.3 and 3.4) that the existence of an extension 
operator (respectively, a norm-preserving extension operator) is sufficient and necessary for projective tensor products to respect their subspace structure isomorphically (respectively, isometrically). This improves a result due to Grothendieck [7, Chapter I, p. 40] where $Y$ was assumed to be complemented in its bidual $Y^{* *}$ (see Remark 3.5).

Section 4 contains the proof of Theorem 1.1. We have tried to give a self-contained proof with as few prerequisites as possible. It turns out (see Remark 4.1) that our proof is simpler than the existing proofs for the above-mentioned special case when $Z=Y^{* *}$.

The notation we use is standard. We consider Banach spaces over the same, either real or complex, field. Let $X$ and $Y$ be Banach spaces. We denote by $\mathcal{L}(X, Y)$ the Banach space of bounded linear operators from $X$ to $Y$ and by $\mathcal{F}(X, Y)$ its linear subspace of finite-rank operators. If $A \in \mathcal{L}(X, Y)$ is an into isomorphism, then its injection modulus $i(A)$ is defined by

$$
i(A)=\sup \{\tau>0:\|A x\| \geqslant \tau\|x\| \forall x \in X\} .
$$

The identity operator on $X$ is denoted by $I_{X}$. We shall always consider $X$ as a subspace of $X^{* *}$, identifying the canonical embedding $j_{X}: X \rightarrow X^{* *}$ with the identity embedding.

\section{Approximation property}

A Banach space $X$ is said to have the approximation property if the identity operator $I_{X}$ on $X$ can be uniformly approximated on compact subsets of $X$ by bounded linear operators of finite rank. In other words, this means that $I_{X}$ belongs to the closure of $\mathcal{F}(X, X)$ in the topology $\tau$ of uniform convergence on compact sets in $X$. Using the description (due to Grothendieck [7]) of the linear functionals on $\mathcal{L}(X, X)$ which are continuous in $\tau$ (see, for example, $[\mathbf{2 0}$, p. 31]), it is easy to show (see, for example, $[\mathbf{2 0}$, p. 32]) that $X$ has the approximation property if and only if the following condition holds.

(AP). For all sequences $\left(x_{n}\right) \subset X$ and $\left(x_{n}^{*}\right) \subset X^{*}$ such that $\sum_{n=1}^{\infty}\left\|x_{n}^{*}\right\|\left\|x_{n}\right\|<\infty$ and $\sum_{n=1}^{\infty} x_{n}^{*}(x) x_{n}=0$, whenever $x \in X$, one has $\sum_{n=1}^{\infty} x_{n}^{*}\left(x_{n}\right)=0$.

Criterion (AP) is one of the most well known from the eight criteria of the approximation property established by Grothendieck in his memoir [7, Chapter I, p. 165], called by him the 'condition de biunivocité'. Several recent criteria of the approximation property may be found in $[\mathbf{1 3}],[\mathbf{1 5}],[\mathbf{1 4}]$ and $[\mathbf{2 3}]$.

By now, it is well known that the approximation property is generally not inherited by subspaces. For instance, the spaces $\ell_{p}, p \neq 2$, and $c_{0}$ are saturated with subspaces which do not have the approximation property: every infinite-dimensional closed subspace of them contains a closed subspace without the approximation property (see, for example, [20, pp. 53 and 90] and [21, p. 107]). Relying on condition (AP), we shall prove the following positive result which is essentially known (see Remark 2.2 below).

Proposition 2.1. Let $Y$ be a closed subspace of a Banach space $Z$. Assume that there is an extension operator $\Phi \in \mathcal{L}\left(Y^{*}, Z^{*}\right)$. If $Z$ has the approximation property, then $Y$ also has the approximation property. 
Proof. Suppose that $Z$ has the approximation property. We shall use condition (AP) to show that $Y$ has the approximation property.

Let $\left(y_{n}\right) \subset Y$ and $\left(y_{n}^{*}\right) \subset Y^{*}$ satisfy $\sum_{n=1}^{\infty}\left\|y_{n}^{*}\right\|\left\|y_{n}\right\|<\infty$ and let $\sum_{n=1}^{\infty} y_{n}^{*}(y) y_{n}=0$ for all $y \in Y$. Then $\sum_{n=1}^{\infty} y^{*}\left(y_{n}\right) y_{n}^{*}=0$ for all $y^{*} \in Y^{*}$, the series being absolutely converging in $Y^{*}$. Therefore,

$$
\sum_{n=1}^{\infty} y^{*}\left(y_{n}\right) \Phi y_{n}^{*}=0 \quad \forall y^{*} \in Y^{*}
$$

meaning that

$$
y^{*}\left(\sum_{n=1}^{\infty}\left(\Phi y_{n}^{*}\right)(z) y_{n}\right)=0 \quad \forall y^{*} \in Y^{*}, \forall z \in Z
$$

Hence

$$
\sum_{n=1}^{\infty}\left(\Phi y_{n}^{*}\right)(z) y_{n}=0 \quad \forall z \in Z
$$

Since $\Phi$ is an extension operator and $Z$ has the approximation property, we have, using condition (AP) for $Z$,

$$
\sum_{n=1}^{\infty} y_{n}^{*}\left(y_{n}\right)=\sum_{n=1}^{\infty}\left(\Phi y_{n}^{*}\right)\left(y_{n}\right)=0 .
$$

Consequently, $Y$ has the approximation property.

Remark 2.2. Kalton [11, Theorem 5.1] and Lima [12, Corollary 2] proved Proposition 2.1 for a locally complemented subspace $Y$ of $Z$. A closed subspace $Y$ of a Banach space $Z$ is called locally complemented in $Z$ if there exists a constant $\lambda \geqslant 1$ such that whenever $F$ is a finite-dimensional subspace of $Z$ and $\varepsilon>0$, there is a linear operator $T: F \rightarrow Y$ with $T x=x$ for all $x \in F \cap Y$ and $\|T\| \leqslant \lambda+\varepsilon$. Kalton considered the separable case and the bounded approximation property, but his argument clearly works also for the general (non-separable) case and for the approximation property, and it was essentially applied by Lima. Let us mention that if $Y$ is locally complemented in $Z$, then, using a compactness argument due to Lindenstrauss [16], one can prove (see [3, Theorem 2.14] or [11, Theorem 3.5]) that there exists an extension operator $\Phi \in \mathcal{L}\left(Y^{*}, Z^{*}\right)$. On the other hand, by the principle of local reflexivity, the converse also holds true (see [3, Theorem 2.14] or [11, Theorem 3.5]).

\section{Projective tensor products and nuclear operators}

Let $X$ and $Y$ be Banach spaces. For the sake of readers who are not acquainted with the theory of tensor products of Banach spaces, let us recall that any element $u=$ $\sum_{n=1}^{m} x_{n} \otimes y_{n}$ of the algebraic tensor product $X \otimes Y$ can be algebraically identified with the finite-rank operator

$$
\sum_{n=1}^{m} x_{n} \otimes y_{n}: x^{*} \mapsto \sum_{n=1}^{m} x^{*}\left(x_{n}\right) y_{n}
$$


from $X^{*}$ to $Y$. Thus $X \otimes Y$ may always be viewed as a linear subspace of $\mathcal{F}\left(X^{*}, Y\right)$. In particular, $X^{*} \otimes Y=\mathcal{F}(X, Y)$.

The class of nuclear operators is closely related to the so-called projective tensor products of Banach spaces. Let us recall that the projective tensor product $X \hat{\otimes} Y$ of Banach spaces $X$ and $Y$ is the completion of the algebraic tensor product $X \otimes Y$ in the (projective or $\pi-)$ norm $\|\cdot\|_{\pi}$ defined as

$$
\|u\|_{\pi}=\inf \left\{\sum_{n=1}^{m}\left\|x_{n}\right\|\left\|y_{n}\right\|: u=\sum_{n=1}^{m} x_{n} \otimes y_{n}\right\}, \quad u \in X \otimes Y,
$$

where the infimum is taken over all possible representations of $u$.

The projective tensor product $X \hat{\otimes} Y$ has a simple description due to Grothendieck [7] (for a proof, we refer, for example, to [2, p. 227]): every $u \in X \hat{\otimes} Y$ has a representation

$$
u=\sum_{n=1}^{\infty} x_{n} \otimes y_{n} \quad \text { with } \sum_{n=1}^{\infty}\left\|x_{n}\right\|\left\|y_{n}\right\|<\infty
$$

(the series is (absolutely) converging for the $\pi$-norm). Moreover,

$$
\|u\|_{\pi}=\inf \left\{\sum_{n=1}^{\infty}\left\|x_{n}\right\|\left\|y_{n}\right\|: u=\sum_{n=1}^{\infty} x_{n} \otimes y_{n}\right\}, \quad u \in X \hat{\otimes} Y,
$$

where the infimum is taken over all representations of $u$ of the form (3.1).

The dual space of a projective tensor product is even easier to describe than the projective tensor product itself. A simple straightforward verification (see, for example, $\left[\mathbf{2}\right.$, pp. 229, 230]) shows that the dual space of $X \hat{\otimes} Y$ can be identified with $\mathcal{L}\left(X, Y^{*}\right)$ or with $\mathcal{L}\left(Y, X^{*}\right)$ under the duality

$$
\left\langle A, \sum_{n=1}^{\infty} x_{n} \otimes y_{n}\right\rangle=\sum_{n=1}^{\infty}\left(A x_{n}\right)\left(y_{n}\right)
$$

or, respectively, under the duality

$$
\left\langle B, \sum_{n=1}^{\infty} x_{n} \otimes y_{n}\right\rangle=\sum_{n=1}^{\infty}\left(B y_{n}\right)\left(x_{n}\right)
$$

This identification is, in fact, a linear isometry. Therefore, one writes

$$
(X \hat{\otimes} Y)^{*}=\mathcal{L}\left(X, Y^{*}\right) \quad \text { or } \quad(X \hat{\otimes} Y)^{*}=\mathcal{L}\left(Y, X^{*}\right) .
$$

The above description was known already to Schatten [31].

Let $X$ be a Banach space and let $Y$ be a closed subspace of a Banach space $Z$. Denote by $j: Y \rightarrow Z$ the identity embedding and consider the identity embedding

$$
I_{X} \otimes j: X \otimes Y \rightarrow X \hat{\otimes} Z
$$


It clearly satisfies

$$
\left\|\left(I_{X} \otimes j\right) u\right\|_{\pi} \leqslant\|u\|_{\pi} .
$$

Let us denote its (unique) bounded extension to $X \hat{\otimes} Y$ also by $I_{X} \otimes j$ and call $I_{X} \otimes j: X \hat{\otimes} Y \rightarrow X \hat{\otimes} Z$ the natural inclusion.

It is well known (see, for example, [2, pp. 230, 231]) that the natural inclusion $I_{X} \otimes$ $j$ need not be isometric: the projective tensor products do not respect the subspace structure. The natural inclusion need not even be isomorphic (see [7, Chapter I, p. 40]; for a stronger result in this direction, see [33, Theorem V.1]). However, as can be seen from the next result, the natural inclusion is an into isomorphism whenever an extension operator exists.

Theorem 3.1. Let $X$ be a Banach space. Let $Y$ be a closed subspace of a Banach space $Z$ and let $j: Y \rightarrow Z$ denote the identity embedding.

(a) If there exists an extension operator $\Phi \in \mathcal{L}\left(Y^{*}, Z^{*}\right)$, then the natural inclusion $I_{X} \otimes j: X \hat{\otimes} Y \rightarrow X \hat{\otimes} Z$ is an into isomorphism satisfying the inequalities

$$
\frac{1}{\|\Phi\|}\|u\|_{\pi} \leqslant\left\|\left(I_{X} \otimes j\right) u\right\|_{\pi} \leqslant\|u\|_{\pi} \quad \forall u \in X \hat{\otimes} Y
$$

(b) If the natural inclusion $I_{Y^{*}} \otimes j: Y^{*} \hat{\otimes} Y \rightarrow Y^{*} \hat{\otimes} Z$ is an into isomorphism, then there exists an extension operator $\Phi \in \mathcal{L}\left(Y^{*}, Z^{*}\right)$ with $\|\Phi\|=1 / i\left(I_{Y^{*}} \otimes j\right)$.

Proof. (a) Consider any $u=\sum_{n=1}^{\infty} x_{n} \otimes y_{n} \in X \hat{\otimes} Y$. One need only verify that

$$
\|u\|_{\pi} \leqslant\|\Phi\|\left\|\left(I_{X} \otimes j\right) u\right\|_{\pi} .
$$

Since $(X \hat{\otimes} Y)^{*}=\mathcal{L}\left(X, Y^{*}\right)$, there exists an operator $A \in \mathcal{L}\left(X, Y^{*}\right)$ with $\|A\|=1$ so that

$$
\|u\|_{\pi}=\langle A, u\rangle=\sum_{n=1}^{\infty}\left(A x_{n}\right)\left(y_{n}\right)=\sum_{n=1}^{\infty}\left(\Phi\left(A x_{n}\right)\right)\left(y_{n}\right) .
$$

On the other hand, since $\Phi A \in \mathcal{L}\left(X, Z^{*}\right)=(X \hat{\otimes} Z)^{*}$, we have

$$
\begin{aligned}
\sum_{n=1}^{\infty}\left(\Phi\left(A x_{n}\right)\right)\left(y_{n}\right) & =\left\langle\Phi A,\left(I_{X} \otimes j\right) u\right\rangle \leqslant\|\Phi A\|\left\|\left(I_{X} \otimes j\right) u\right\|_{\pi} \\
& \leqslant\|\Phi\|\left\|\left(I_{X} \otimes j\right) u\right\|_{\pi} .
\end{aligned}
$$

This yields the desired inequality.

(b) Using the description of duals of projective tensor products, let us consider

$$
\left(I_{Y^{*}} \otimes j\right)^{*}: \mathcal{L}\left(Y^{*}, Z^{*}\right) \rightarrow \mathcal{L}\left(Y^{*}, Y^{*}\right) .
$$

Since $I_{Y^{*}} \otimes j$ is an into isomorphism, there exists a $\Phi \in \mathcal{L}\left(Y^{*}, Z^{*}\right)$ so that $\left(I_{Y^{*}} \otimes j\right)^{*} \Phi=$ $I_{Y^{*}}$ and

$$
\|\Phi\| \leqslant \frac{1}{i\left(I_{Y^{*}} \otimes j\right)}\left\|I_{Y^{*}}\right\|=\frac{1}{i\left(I_{Y^{*}} \otimes j\right)}
$$


(for a proof of this 'folkloristic' fact, see, for example, [8, Lemma 2.2]). Since, for all $y^{*} \in Y^{*}$ and $y \in Y$,

$$
\left(\Phi y^{*}\right)(y)=\left\langle\Phi, y^{*} \otimes y\right\rangle=\left\langle\left(I_{Y^{*}} \otimes j\right)^{*} \Phi, y^{*} \otimes y\right\rangle=\left\langle I_{Y^{*}}, y^{*} \otimes y\right\rangle=y^{*}(y)
$$

$\Phi$ is an extension operator. By the already-proved part (a), we also have $i\left(I_{Y^{*}} \otimes j\right) \geqslant$ $1 /\|\Phi\|$.

Remark 3.2. If $\|\Phi\|=1$ in Theorem 3.1 (a), that is, if $\Phi$ is a norm-preserving extension operator, then $I_{X} \otimes j$ is an isometry and $X \hat{\otimes} Y$ is a subspace of $X \hat{\otimes} Z$ (this result was proved by Randrianantoanina [27] and Rao [28]). In particular, if $Z=Y^{* *}$ and $\Phi=j_{Y^{*}}$, one has the well-known result due to Grothendieck [7] that $X \hat{\otimes} Y$ is a subspace of $X \hat{\otimes} Y^{* *}$. The special case of Theorem 3.1 (a), when $Y$ is complemented in $Z$ (observe that if $P \in \mathcal{L}(Z, Y)$ is a projection onto $Y$, then $P^{*}$ is clearly an extension operator), is well known (see [7, Chapter I, p. 40] or, for example, [30, p. 18]).

The following characterizations of the situations when the projective tensor products respect the subspace structure isomorphically or isometrically are immediate from Theorem 3.1 .

Theorem 3.3. Let $Y$ be a closed subspace of a Banach space $Z$. Then the following assertions are equivalent.

(a) The natural inclusion from $X \hat{\otimes} Y$ to $X \hat{\otimes} Z$ is an into isomorphism for all Banach spaces $X$.

(b) The natural inclusion from $Y^{*} \hat{\otimes} Y$ to $Y^{*} \hat{\otimes} Z$ is an into isomorphism.

(c) There exists an extension operator $\Phi \in \mathcal{L}\left(Y^{*}, Z^{*}\right)$.

Moreover, in this case, $i\left(I_{X} \otimes j\right) \geqslant i\left(I_{Y^{*}} \otimes j\right)$, for all Banach spaces $X$, and

$$
i\left(I_{Y^{*}} \otimes j\right)=\max \left\{1 /\|\Phi\|: \Phi \in \mathcal{L}\left(Y^{*}, Z^{*}\right) \text { is an extension operator }\right\}
$$

where $j$ denotes the identity embedding from $Y$ to $Z$.

Theorem 3.4. Let $Y$ be a closed subspace of a Banach space $Z$. Then the following assertions are equivalent.

(a) $X \hat{\otimes} Y$ is a closed linear subspace of $X \hat{\otimes} Z$ (under the natural inclusion) for all Banach spaces $X$.

(b) $Y^{*} \hat{\otimes} Y$ is a closed linear subspace of $Y^{*} \hat{\otimes} Z$ (under the natural inclusion).

(c) There exists a linear norm-preserving extension operator from $Y^{*}$ to $Z^{*}$.

Remark 3.5. Theorem 3.3, together with Theorem 3.4, improves the following result due to Grothendieck [7, Chapter I, p. 40].

Let $Y$ be a closed subspace of a Banach space $Z$ such that $Y$ is complemented in its bidual $Y^{* *}$. Then the natural inclusion from $Y^{*} \hat{\otimes} Y$ to $Y^{*} \hat{\otimes} Z$ is an into isomorphism if and only if $Y$ is complemented in $Z$. 
To see that Theorem 3.3 contains Grothendieck's result as a particular case, let us observe that if $P \in \mathcal{L}\left(Y^{* *}, Y\right)$ is a projection onto $Y$ and if $\Phi \in \mathcal{L}\left(Y^{*}, Z^{*}\right)$ is an extension operator, then, clearly, $\left.\Phi^{*}\right|_{Y}=I_{Y}$ and therefore $\left.P \Phi^{*}\right|_{Z}$ is a projection from $Z$ onto $Y$. On the other hand, if $Q \in \mathcal{L}(Z, Y)$ is a projection onto $Y$, then $Q^{*}$ is clearly an extension operator.

Let $X$ and $Y$ be Banach spaces. Then there is a natural linear surjection from $X^{*} \hat{\otimes} Y$ onto $\mathcal{N}(X, Y)$. It assigns to any $u \in X^{*} \hat{\otimes} Y$, having a representation

$$
u=\sum_{n=1}^{\infty} x_{n}^{*} \otimes y_{n} \quad \text { with } \sum_{n=1}^{\infty}\left\|x_{n}^{*}\right\|\left\|y_{n}\right\|<\infty,
$$

the nuclear operator

$$
\sum_{n=1}^{\infty} x_{n}^{*} \otimes y_{n}: x \mapsto \sum_{n=1}^{\infty} x_{n}^{*}(x) y_{n} .
$$

If now $X^{*}$ or $Y$ has the approximation property, then using the description of $\left(X^{*} \hat{\otimes} Y\right)^{*}$ as $\mathcal{L}\left(Y, X^{* *}\right)$ or, respectively, $\mathcal{L}\left(X^{*}, Y^{*}\right)$ together with the Hahn-Banach theorem, and relying on condition (AP), it is rather straightforward to verify that the natural surjection above is also injective (cf. [7, Chapter I, p. 167]). In fact, it is a linear isometry between $X^{*} \hat{\otimes} Y$ and $\mathcal{N}(X, Y)$ (this is obvious from the definitions of the norms $\|\cdot\|_{\pi}$ and $\|\cdot\|_{\mathcal{N}}$ ). Therefore, one writes

$$
X^{*} \hat{\otimes} Y=\mathcal{N}(X, Y)
$$

whenever $X^{*}$ or $Y$ has the approximation property, identifying $X^{*} \hat{\otimes} Y$ and $\mathcal{N}(X, Y)$ as Banach spaces.

For the proof of our main Theorem 1.1, we shall need the following corollary of Proposition 2.1 and Theorem 3.1 (a).

Proposition 3.6. Let $X$ be a Banach space. Let $Y$ be a closed subspace of a Banach space $Z$ and let $j: Y \rightarrow Z$ denote the identity embedding. Assume that there is an extension operator $\Phi \in \mathcal{L}\left(Y^{*}, Z^{*}\right)$. If $X^{*}$ or $Z$ has the approximation property, then, for every nuclear operator $T \in \mathcal{N}(X, Y)$, the following inequalities hold:

$$
\frac{1}{\|\Phi\|}\|T\|_{\mathcal{N}} \leqslant\|j T\|_{\mathcal{N}} \leqslant\|T\|_{\mathcal{N}}
$$

Proof. First of all, notice that $Y$ also has the approximation property (see Proposition 2.1). Let a nuclear representation of $T$ be given by

$$
u \in X^{*} \hat{\otimes} Y, \quad u=\sum_{n=1}^{\infty} x_{n}^{*} \otimes y_{n}, \quad \sum_{n=1}^{\infty}\left\|x_{n}^{*}\right\|\left\|y_{n}\right\|<\infty .
$$

Then a nuclear representation of $j T$ is given by $\left(I_{X^{*}} \otimes j\right) u=\sum_{n=1}^{\infty} x_{n}^{*} \otimes j y_{n} \in X^{*} \hat{\otimes} Z$. Since $X^{*}$ or $Y$ has the approximation property, $\|T\|_{\mathcal{N}}=\|u\|_{\pi}$. Since $X^{*}$ or $Z$ has the approximation property, $\|j T\|_{\mathcal{N}}=\left\|\left(I_{X^{*}} \otimes j\right) u\right\|_{\pi}$. And the required inequalities are obvious from Theorem 3.1 (a). 


\section{Proof of Theorem 1.1}

First of all, notice that $Z$ also has the approximation property if $Z^{*}$ does (this is a wellknown result due to Grothendieck [7] which is obvious from condition (AP)). Therefore, relying on Proposition 3.6, one need only prove that $T \in \mathcal{N}(X, Y)$ whenever $T \in \mathcal{L}(X, Y)$ and $j T \in \mathcal{N}(X, Z)$. In other words, one need prove that $j T$ belongs to the subspace $\{j S: S \in \mathcal{N}(X, Y)\}$ of $\mathcal{N}(X, Z)$. This subspace, being isomorphic to $\mathcal{N}(X, Y)$ (by Proposition 3.6), is a closed subspace of $\mathcal{N}(X, Z)$. Therefore, it suffices to show that every continuous linear functional on $\mathcal{N}(X, Z)$ that vanishes on $\left\{j S: S=x^{*} \otimes y: x^{*} \in\right.$ $\left.X^{*}, y \in Y\right\}$, also vanishes on $j T$. Let $j T=\sum_{n=1}^{\infty} x_{n}^{*} \otimes z_{n}$ with $x_{n}^{*} \in X^{*}, z_{n} \in Z$, and $\sum_{n=1}^{\infty}\left\|x_{n}^{*}\right\|\left\|z_{n}\right\|<\infty$.

(1) Assume that $X^{*}$ has the approximation property and use the canonical identifications

$$
\mathcal{N}(X, Z)^{*}=\left(X^{*} \hat{\otimes} Z\right)^{*}=\mathcal{L}\left(Z, X^{* *}\right) .
$$

Suppose that $A \in \mathcal{L}\left(Z, X^{* *}\right)$ satisfies

$$
\left\langle A, j\left(x^{*} \otimes y\right)\right\rangle=0 \quad \forall x^{*} \in X^{*}, \forall y \in Y .
$$

This means that $(A j y)\left(x^{*}\right)=0$ for all $x^{*} \in X^{*}$ and all $y \in Y$, or $A j=0$ as an operator from $Y$ to $X^{* *}$.

The desired equality

$$
\langle A, j T\rangle=\sum_{n=1}^{\infty}\left(A z_{n}\right)\left(x_{n}^{*}\right)=0
$$

follows from condition (AP) for $X^{*}$, because, for all $x^{*} \in X^{*}$ and $x \in X$,

$$
\begin{aligned}
\left(\sum_{n=1}^{\infty}\left(A z_{n}\right)\left(x^{*}\right) x_{n}^{*}\right)(x) & =\left(\sum_{n=1}^{\infty} x_{n}^{*}(x) A z_{n}\right)\left(x^{*}\right)=((A j T) x)\left(x^{*}\right) \\
& =((0 T) x)\left(x^{*}\right)=0 .
\end{aligned}
$$

(2) Assume that $Z^{*}$ has the approximation property and use the canonical identifications

$$
\mathcal{N}(X, Z)^{*}=\left(X^{*} \hat{\otimes} Z\right)^{*}=\mathcal{L}\left(X^{*}, Z^{*}\right) .
$$

Suppose that $A \in \mathcal{L}\left(X^{*}, Z^{*}\right)$ satisfies

$$
\left\langle A, j\left(x^{*} \otimes y\right)\right\rangle=0 \quad \forall x^{*} \in X^{*}, \forall y \in Y .
$$

This means that $\left(A x^{*}\right)(j y)=\left(j^{*} A x^{*}\right)(y)=0$ for all $x^{*} \in X^{*}$ and $y \in Y$, or $j^{*} A=0$ as an operator from $X^{*}$ to $Y^{*}$.

To establish the desired equality

$$
\langle A, j T\rangle=\sum_{n=1}^{\infty}\left(A x_{n}^{*}\right)\left(z_{n}\right)=0,
$$


let us observe that, for all $n$,

$$
\left(j^{* *} \Phi^{*} z_{n}\right)\left(A x_{n}^{*}\right)=\left(\Phi^{*} z_{n}\right)\left(j^{*} A x_{n}^{*}\right)=\left(\Phi^{*} z_{n}\right)(0)=0 .
$$

Consequently,

$$
\langle A, j T\rangle=\sum_{n=1}^{\infty}\left(z_{n}-j^{* *} \Phi^{*} z_{n}\right)\left(A x_{n}^{*}\right) .
$$

Therefore, the equality $\langle A, j T\rangle=0$ follows from condition (AP) for $Z^{*}$, because, for all $z^{*} \in Z^{*}$, we have

$$
\begin{aligned}
\sum_{n=1}^{\infty}\left(z_{n}-j^{* *} \Phi^{*} z_{n}\right)\left(z^{*}\right) A x_{n}^{*} & =A\left(\sum_{n=1}^{\infty} z_{n}\left(z^{*}\right) x_{n}^{*}-\sum_{n=1}^{\infty} z_{n}\left(\Phi j^{*} z^{*}\right) x_{n}^{*}\right) \\
& =A\left((j T)^{*}\left(z^{*}-\Phi j^{*} z^{*}\right)\right)=\left(A T^{*}\right)\left(j^{*} z^{*}-j^{*} \Phi j^{*} z^{*}\right) \\
& =\left(A T^{*}\right)\left(j^{*} z^{*}-j^{*} z^{*}\right)=0
\end{aligned}
$$

Remark 4.1. If one carried out the proof of Theorem 1.1 in the well-known particular case (due to Grothendieck [7]) when $Z=Y^{* *}$ with $\Phi=j_{Y^{*}}$, and $X^{*}$ has the approximation property, then one would observe that our argument is simpler and shorter than the traditional ones (cf. [7, Chapter I, pp. 85, 86], [2, p. 243] or [30, p. 77]). If one carried out our proof in the other known particular case (see [24] or [25]) when $Z=Y^{* *}$ with $\Phi=j_{Y^{*}}$, and $Y^{* * *}$ has the approximation property, then one would observe that it is much simpler and shorter than the proof in [25]. The main difference is that the above-mentioned proofs rely on derivations of condition (AP), whereas our proof makes a direct use of this condition. We have also been trying to reduce to a minimum the use of tensor products machinery.

\section{Comments and applications}

The notation will be as above. In particular, $X$ is a Banach space, $Y$ is a closed subspace of a Banach space $Z$, and $j: Y \rightarrow Z$ denotes the identity embedding.

Theorem 1.1 shows that if $Y$ is sufficiently well placed in $Z$-more precisely, if there exists an extension operator $\Phi \in \mathcal{L}\left(Y^{*}, Z^{*}\right)$-and if $X^{*}$ or $Z^{*}$ has the approximation property, then

$$
T \in \mathcal{L}(X, Y) \text { and } j T \in \mathcal{N}(X, Z) \Rightarrow T \in \mathcal{N}(X, Y) .
$$

\section{1 .}

As was already mentioned in $\S 1$, Theorem 1.1 contains the Grothendieck-Oja-Reinov result as an immediate particular case with $Z=Y^{* *}$ and $\Phi=j_{Y^{*}}$. In this case, and therefore also in Theorem 1.1, the assumptions about the approximation properties of $X^{*}$ and $Z^{*}$ are essential and cannot be weakened to the approximation properties of $X$ or/and $Z$ (or even to the existence of bases). In fact, as it was shown by Oja and Reinov 
(using the famous James-Lindenstrauss construction [17]) (see $[\mathbf{2 4}, \mathbf{2 5}]$ ), there exists a Banach space $Y$ such that $Y^{* *}$ has a boundedly complete basis, $Y^{* * *}$ is separable but it does not have the approximation property, and there exists an operator $T \in \mathcal{L}\left(Y^{* *}, Y\right)$ so that $j_{Y} T \in \mathcal{N}\left(Y^{* *}, Y^{* *}\right)$ but $T \notin \mathcal{N}\left(Y^{* *}, Y\right)$. In this example, $X=Z=Y^{* *}$. Let us notice that the need for the approximation property of $X^{*}$ is already clear from the important paper by Figiel and Johnson [4] from 1973.

\section{2 .}

Let us now show that if $\left(2^{\prime}\right)$ holds for $X=Y$ such that $X^{*}=Y^{*}$ has the approximation property, then necessarily an extension operator $\Phi \in \mathcal{L}\left(Y^{*}, Z^{*}\right)$ must exist.

First of all observe that

$$
W:=\{j T: T \in \mathcal{L}(X, Y) \text { and } j T \in \mathcal{N}(X, Z)\}
$$

is a closed subspace of $\mathcal{N}(X, Z)$ containing

$$
V:=\{j S: S \in \mathcal{N}(X, Y)\}
$$

The natural embedding $J: \mathcal{N}(X, Y) \rightarrow \mathcal{N}(X, Z)$, given by $J(S)=j S, S \in \mathcal{N}(X, Y)$, is clearly injective and $\operatorname{ran} J=V$. Observe also that $\left(2^{\prime}\right)$ holds if and only if $V=W$.

Therefore, if $\left(2^{\prime}\right)$ holds, then $J$ is an into isomorphism, and we may use the following immediate corollary of Theorem 3.3.

Proposition 5.1. If $Y^{*}$ has the approximation property, then the following assertions are equivalent.

(a) The natural embedding $J: \mathcal{N}(Y, Y) \rightarrow \mathcal{N}(Y, Z)$ is an into isomorphism.

(b) There exists an extension operator $\Phi \in \mathcal{L}\left(Y^{*}, Z^{*}\right)$.

Moreover, in this case, $i(J)=\max \left\{1 /\|\Phi\|: \Phi \in \mathcal{L}\left(Y^{*}, Z^{*}\right)\right.$ is an extension operator $\}$.

\section{3 .}

Implication $\left(2^{\prime}\right)$ holds for arbitrary Banach spaces $X$ whenever $Y$ is a complemented subspace of $Z$. Indeed, let $P \in \mathcal{L}(Z, Y)$ be a projection onto $Y$. If $T \in \mathcal{L}(X, Y)$ and $j T \in \mathcal{N}(X, Z)$, then $T=P j T \in \mathcal{N}(X, Y)$ (and, moreover, $\|T\|_{\mathcal{N}} \leqslant\|P\|\|j T\|_{\mathcal{N}}$ ).

On the other hand, $Y$ is complemented in $Z$ whenever $Y$ is complemented in its bidual $Y^{* *}, Y^{*}$ has the approximation property, and $J: \mathcal{N}(Y, Y) \rightarrow \mathcal{N}(Y, Z)$ is an into isomorphism. (This result, which is due to Grothendieck, easily follows from Proposition 5.1 (see Remark 3.5).)

Therefore, considering uncomplemented subspaces $Y$ of $Z$, one can give numerous examples of situations when $\left(2^{\prime}\right)$ does not hold despite the fact that $X, Y, Z$ and all their higher duals have the approximation property. For instance, $\left(2^{\prime}\right)$ does not hold for $X=Y$ being an isomorphic copy of $\ell_{2}$ in $Z=\ell_{\infty}$. Similar examples may be obtained using, for example, uncomplemented subspaces isomorphic to $\ell_{1}$ in $C[0,1]$ (classical Banach-Mazur example) or to $\ell_{2}$ in $L_{4 / 3}[0,1]$ (example due to Rosenthal $[\mathbf{2 9}$, p. 52]). 
Stegall and Retherford [33, p. 475] essentially showed that $\left(2^{\prime}\right)$ fails for Rosenthal's example, and also if $X=\ell_{2}$ and $Y$ is a sufficiently Euclidean subspace, with a basis, of $Z=\ell_{1}$. In the last case, they used results established in [33] and a simple wellknown fact (see, for example, $[\mathbf{3 5}$, p. 126]) (that can also be used for the examples of the latter paragraph): if $X^{*}$ has the approximation property, then the natural embedding $J: \mathcal{N}(X, Y) \rightarrow \mathcal{N}(X, Z)$ is an into isomorphism if and only if the restriction mapping from $\mathcal{L}\left(Z, X^{* *}\right)$ to $\mathcal{L}\left(Y, X^{* *}\right)$ given by $\left.A \mapsto A\right|_{Y}$ (which actually coincides with $J^{*}$ ) is surjective.

\section{4 .}

In contract with 5.3 above, let us observe the following result, which is essentially due to Stegall and Retherford [33]. For the definition and basic properties of $\mathcal{L}_{\infty}$-spaces (or, more generally, $\mathcal{L}_{p}$-spaces), we refer to $[\mathbf{1 8}]$ and $[\mathbf{1 9}]$.

Theorem 5.2. Implication $\left(2^{\prime}\right)$ holds for arbitrary Banach spaces $Z$ and their closed subspaces $Y$ if and only if $X$ is an $\mathcal{L}_{\infty}$-space.

Proof. By [33, Theorem III.3], $X$ is an $\mathcal{L}_{\infty}$-space if and only if for any Banach space $Z$ and for any $T \in \mathcal{N}(X, Z)$, the astriction $T_{a}: X \rightarrow \overline{\operatorname{ran} T}$ is a nuclear operator. From this, our claim is straightforward.

\section{5 .}

There are many important situations that are different, in general, from the classical one considered in 5.1, when an extension operator $\Phi \in \mathcal{L}\left(Y^{*}, Z^{*}\right)$ exists and Theorem 1.1 applies.

\subsection{1.}

Recall that a Banach space is said to be a $\mathcal{P}_{\lambda^{-}}$space, for some $\lambda \geqslant 1$, if it is complemented, by a projection whose norm does not exceed $\lambda$, in any Banach space containing it (as an isometrically isomorphic subspace). Recall also that, for every set $\Gamma$, the space $\ell_{\infty}(\Gamma)$ is a $\mathcal{P}_{1}$-space (see, for example, $[\mathbf{2 0}$, p. 105]).

The next result is due to Fakhoury [3, Corollary 3.3]. Fakhoury's proof relies on Lindenstrauss's memoir [16] and his own results established in [3]. We present a simple direct proof.

Proposition 5.3. Let $Y$ be a closed subspace of a Banach space $Z$. If $Y^{* *}$ is a $\mathcal{P}_{\lambda^{-}}$ space, then there exists an extension operator $\Phi \in \mathcal{L}\left(Y^{*}, Z^{*}\right)$ with $\|\Phi\| \leqslant \lambda$.

Proof. Since $j^{* *}: Y^{* *} \rightarrow Z^{* *}$ is an into isometry, there exists an operator $P \in$ $\mathcal{L}\left(Z^{* *}, Y^{* *}\right)$ with $\|P\| \leqslant \lambda$ such that $P j^{* *} y^{* *}=y^{* *}$ for all $y^{* *} \in Y^{* *}$. Put $\Phi=j_{Z}^{*} P^{*} j_{Y^{*}}$. Since, for all $y^{*} \in Y^{*}$ and $y \in Y$,

$$
\left(\Phi y^{*}\right)(j y)=\left(j_{Y^{*}} y^{*}\right)\left(P j_{Z} j y\right)=\left(j_{Y^{*}} y^{*}\right)\left(P j^{* *} j_{Y} y\right)=\left(j_{Y^{*}} y^{*}\right)\left(j_{Y} y\right)=y^{*}(y)
$$

$\Phi$ is an extension operator. 
Proposition 5.3 gives access to an immediate application of Theorem 1.1. However, in this case, it is possible to avoid the approximation conditions imposed on $X^{*}$ or $Z^{*}$.

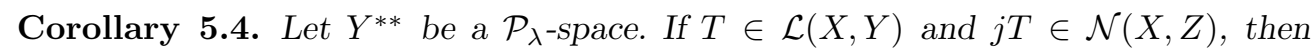
$T \in \mathcal{N}(X, Y)$ and

$$
\frac{1}{\lambda}\|T\|_{\mathcal{N}} \leqslant\|j T\|_{\mathcal{N}} \leqslant\|T\|_{\mathcal{N}}
$$

Proof. Let $\Gamma$ be a set such that there exists an into isometry $i: Z \rightarrow \ell_{\infty}(\Gamma)$. Since $j T$ is nuclear, we have that $i j T \in \mathcal{N}\left(X, \ell_{\infty}(\Gamma)\right)$. Because of Proposition 5.3, and since $\ell_{\infty}(\Gamma)^{*}$ has the (metric) approximation property, Theorem 1.1 applies. Hence, $T \in \mathcal{N}(X, Y)$ and

$$
\frac{1}{\lambda}\|T\|_{\mathcal{N}} \leqslant\|i j T\|_{\mathcal{N}} \leqslant\|j T\|_{\mathcal{N}} \leqslant\|T\|_{\mathcal{N}}
$$

Recall that the Banach-Mazur distance of two isomorphic Banach spaces $X$ and $Y$ is defined as $d(X, Y)=\inf \left\{\|\varphi\|\left\|\varphi^{-1}\right\|: \varphi\right.$ is an isomorphism from $X$ onto $\left.Y\right\}$.

Corollary 5.5. Let a subspace $Y$ of a Banach space $Z$ be isomorphic to $c_{0}(\Gamma)$, for some set $\Gamma$. If $T \in \mathcal{L}(X, Y)$ and $j T \in \mathcal{N}(X, Z)$, then $T \in \mathcal{N}(X, Y)$ and

$$
\frac{1}{d\left(Y, c_{0}(\Gamma)\right)}\|T\|_{\mathcal{N}} \leqslant\|j T\|_{\mathcal{N}} \leqslant\|T\|_{\mathcal{N}} .
$$

Proof. Using that $c_{0}(\Gamma)^{* *}=\ell_{\infty}(\Gamma)$ is a $\mathcal{P}_{1}$-space, it is straightforward to verify that

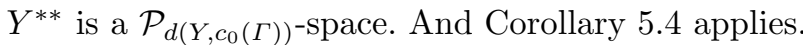

Concerning Corollary 5.5, let us mention that $d\left(Y, c_{0}\right)=1$ implies that $Y$ is isometrically isomorphic to $c_{0}$ (this is proved in [9, p. 120], using $M$-ideal arguments).

It is well known that if $Y$ is an $\mathcal{L}_{\infty}$-space, then $Y^{* *}$ is a $\mathcal{P}_{\lambda}$-space for some $\lambda \geqslant 1$. (This clearly follows from Lindenstrauss's memoir [16]. (In fact, if $Y$ is an $\mathcal{L}_{\infty}$-space, then, by definition, $Y$ is an $\mathcal{L}_{\infty, \lambda}$-space for some $\lambda \geqslant 1$, easily implying that $Y$ is an $\mathcal{N}_{\lambda}$-space

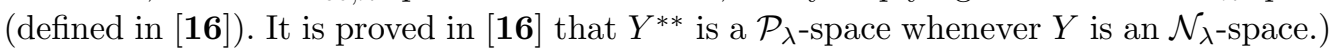
Alternatively, this is also proved in [19, p. 335], relying on [18].) Therefore, Corollary 5.4 immediately yields the following.

Corollary 5.6. If $Y$ is an $\mathcal{L}_{\infty}$-space, then $\left(2^{\prime}\right)$ holds for arbitrary Banach spaces $X$ and $Z$ whenever $Z$ contains $Y$.

The special case of Corollary 5.6 with $Z=\ell_{\infty}(\Gamma)$ may be restated in terms of quasinuclear operators [26] as follows. All quasi-nuclear operators from any Banach space $X$ to any $\mathcal{L}_{\infty}$-space $Y$ are nuclear. This result is due to Stegall and Retherford (see the theorem on p. 480 of $[\mathbf{3 3}])$. 


\subsection{2.}

Let $E$ and $F$ be Banach spaces and let $\mathcal{K}(E, F)$ denote the subspace of compact operators of $\mathcal{L}(E, F)$. Assume that $E^{*}$ or $F$ has the $\lambda$-bounded approximation property for some $\lambda \geqslant 1$. (Recall that this means that the finite-rank operators in the definition of the approximation property can be chosen so that their norms do not exceed $\lambda$. Recall also that most of the common Banach spaces have the 1-bounded (or metric) approximation property.) In this case, an extension operator $\Phi \in \mathcal{L}\left(\mathcal{K}(E, F)^{*}, \mathcal{L}(E, F)^{*}\right)$ with $\|\Phi\| \leqslant \lambda$ can be constructed more or less explicitly (as it was by Johnson in the proof of Lemma 1 in $[\mathbf{1 0}]$ ). And the following is immediate from Theorem 1.1.

Corollary 5.7. Let $X^{*}$ have the approximation property and let $E^{*}$ or $F$ have the $\lambda$-bounded approximation property. If $T \in \mathcal{L}(X, \mathcal{K}(E, F))$ and $j T \in \mathcal{N}(X, \mathcal{L}(E, F))$, then $T \in \mathcal{N}(X, \mathcal{K}(E, F))$ and

$$
\frac{1}{\lambda}\|T\|_{\mathcal{N}} \leqslant\|j T\|_{\mathcal{N}} \leqslant\|T\|_{\mathcal{N}}
$$

We did not dare spell out the version of Corollary 5.7 under the hypothesis that $\mathcal{L}(E, F)^{*}$ had the approximation property since, for instance, by a well-known result of Szankowski $[\mathbf{3 4}], \mathcal{L}\left(\ell_{2}, \ell_{2}\right)$ already fails to have the approximation property.

\subsection{3.}

Sims and Yost [32] have proved that every separable subspace of a (non-separable) Banach space $Z$ is contained in a separable closed subspace $Y$ which admits a normpreserving extension operator $\Phi \in \mathcal{L}\left(Y^{*}, Z^{*}\right)$. From this and Theorem 1.1, the following is immediate.

Corollary 5.8. Let $X^{*}$ or $Z^{*}$ have the approximation property. Then every separable subspace of $Z$ is contained in a closed separable subspace $Y$ of $Z$ with the following property: if $T \in \mathcal{L}(X, Y)$ and $j T \in \mathcal{N}(X, Z)$, then $T \in \mathcal{N}(X, Y)$ and $\|T\|_{\mathcal{N}}=\|j T\|_{\mathcal{N}}$.

\subsection{4.}

It is well known and easy to verify that an extension operator $\Phi \in \mathcal{L}\left(Y^{*}, Z^{*}\right)$ exists if and only if the annihilator $Y^{\perp}$ coincides with the kernel of a bounded linear projection $P$ on $Z^{*}$. In this case, $\|\Phi\|=\|P\|$. If, moreover, $\left\|P z^{*}\right\|+\left\|z^{*}-P z^{*}\right\|=\left\|z^{*}\right\|$ for all $z^{*} \in Z^{*}$, then $Y$ is called an $M$-ideal. There is an extensive literature dealing with $M$-ideals. In particular, $M$-ideals have been described in many important classes of Banach spaces (e.g. in the space $A(K)$ of real-valued affine continuous functions on a compact convex set; in the space $C_{0}(S)$ of continuous functions on a locally compact Hausdorff space $S$ vanishing at infinity; in the disc algebra; in function algebras on a compact space $K$; in unital commutative Banach algebras; in $C^{*}$-algebras; in injective tensor products) (see the monograph [9] for results and references). Theorem 1.1 applies in all those situations. Let us conclude the paper with a couple of such applications. 
Corollary 5.9. Let $C(K)$ denote the space of continuous functions on a compact space $K$. Let $D$ be a closed subset of $K$ and let $Y=\{x \in C(K): x(t)=0$ for all $t \in D\}$. If $T \in \mathcal{L}(X, Y)$ and $j T \in \mathcal{N}(X, C(K))$, then $T \in \mathcal{N}(X, Y)$ and $\|T\|_{\mathcal{N}}=\|j T\|_{\mathcal{N}}$.

In Corollary 5.9, we used the well-known fact that $C(K)^{*}$ (actually any higher dual of $C(K))$ has the (metric) approximation property.

Corollary 5.10. Let $Z$ be a $C^{*}$-algebra and let $Y$ be a closed two-sided ideal of $Z$. Assume that $X^{*}$ or $Z^{*}$ has the approximation property. If $T \in \mathcal{L}(X, Y)$ and $j T \in$ $\mathcal{N}(X, Z)$, then $T \in \mathcal{N}(X, Y)$ and $\|T\|_{\mathcal{N}}=\|j T\|_{\mathcal{N}}$.

Acknowledgements. This research was partially supported by Estonian Science Foundation under grants 4400 and 5704 .

\section{References}

1. J. DiEstel, Geometry of Banach spaces-selected topics, Lecture Notes in Mathematics, vol. 485 (Springer, 1975).

2. J. Diestel And J. J. Uhl, Vector measures, Mathematical Surveys, vol. 15 (American Mathematical Society, Providence, RI, 1977).

3. H. FAKHOURY, Sélections linéaires associées au théorème de Hahn-Banach, J. Funct. Analysis 11 (1972), 436-452.

4. T. Figiel And W. B. Johnson, The approximation property does not imply the bounded approximation property, Proc. Am. Math. Soc. 41 (1973), 197-200.

5. G. Godefroy, N. J. Kalton and P. D. Saphar, Unconditional ideals in Banach spaces, Studia Math. 104 (1993), 13-59.

6. A. GRothendieck, Résumé de la théorie métrique des produits tensoriels topologiques, Boll. Soc. Mat. São Paulo 8 (1953/1956), 1-79.

7. A. Grothendieck, Produits tensoriels topologiques et espaces nucléaires, Memoirs of the American Mathematical Society, vol. 16 (1955).

8. R. HAller, E. OJA AND E. Plewnia, Quantitative versions of hereditary results on $M$-ideals of compact operators, Math. Nachr. 246-247 (2002), 106-120.

9. P. Harmand, D. Werner And W. Werner, $M$-ideals in Banach spaces and Banach algebras, Lecture Notes in Mathematics, vol. 1547 (Springer, 1993).

10. J. Johnson, Remarks on Banach spaces of compact operators, J. Funct. Analysis 32 (1979), 304-311.

11. N. J. Kalton, Locally complemented subspaces and $\mathcal{L}_{p^{-}}$-spaces for $0<p<1$, Math. Nachr. 115 (1984), 71-97.

12. A. LIMA, The metric approximation property, norm-one projections and intersection properties of balls, Israel J. Math. 84 (1993), 451-475.

13. A. LimA AND E. OJA, Ideals of finite rank operators, intersection properties of balls, and the approximation property, Studia Math. 133 (1999), 175-186.

14. A. LIMA AND E. OJA, Ideals of operators, approximability in the strong operator topology, and the approximation property, Michigan Math. J. 52 (2004), 253-265.

15. A. Lima, O. NyGAARD AND E. OJA, Isometric factorization of weakly compact operators and the approximation property, Israel J. Math. 119 (2000), 325-348.

16. J. Lindenstrauss, Extension of compact operators, Memoirs of the American Mathematical Society, vol. 48 (1964).

17. J. Lindenstrauss, On James's paper 'Separable conjugate spaces', Israel J. Math. 9 (1971), 279-284. 
18. J. Lindenstrauss And A. PęCZYŃski, Absolutely summing operators in $\mathcal{L}_{p}$-spaces and their applications, Studia Math. 29 (1968), 275-326.

19. J. Lindenstrauss and H. P. Rosenthal, The $\mathcal{L}_{p}$ spaces, Israel J. Math. 7 (1969), 325-349.

20. J. Lindenstrauss And L. Tzafriri, Classical Banach spaces, vol. I (Springer, 1977).

21. J. Lindenstrauss and L. Tzafriri, Classical Banach spaces, vol. II (Springer, 1979).

22. E. OJA, Geometry of Banach spaces having shrinking approximations of the identity, Trans. Am. Math. Soc. 352 (2000), 2801-2823.

23. E. OJA AND A. PELANDER, The approximation property in terms of the approximability of weak*-weak continuous operators, J. Math. Analysis Applic. 286 (2003), 713-723.

24. E. OJa And O. Reinov, Un contre-exemple à une affirmation de A. Grothendieck, $C . R$. Acad. Sci. Paris Sér. I 305 (1987), 121-122.

25. E. Oja And O. Reinov, A counterexample to A. Grothendieck, Proc. Acad. Sci. Estonian SSR Phys. Math. 37 (1988), 14-17 (in Russian with Estonian and English summaries).

26. A. Pietsch, Quasinukleare Abbildungen in normierten Räumen, Math. Annln 165 (1966), 76-90.

27. N. Randrianantoanina, Complemented copies of $\ell^{1}$ and Pełczyński's property $\left(V^{*}\right)$ in Bochner function spaces, Can. J. Math. 48 (1996), 625-640.

28. T. S. S. R. K. RAO, On ideals in Banach spaces, Rocky Mt. J. Math. 31 (2001), 595-609.

29. H. P. Rosenthal, Projections onto translation-invariant subspaces of $L^{p}(G)$, Memoirs of the American Mathematical Society, vol. 63 (1966).

30. R. A. RYAN, Introduction to tensor products of Banach spaces (Springer, 2002).

31. R. Schatten, The cross-space of linear transformations, Ann. Math. 47 (1946), 73-84.

32. B. Sims And D. Yost, Linear Hahn-Banach extension operators, Proc. Edinb. Math. Soc. 32 (1989), 53-57.

33. C. P. Stegall And J. R. Retherford, Fully nuclear and completely nuclear operators with applications to $\mathcal{L}_{1-}$ and $\mathcal{L}_{\infty}$-spaces, Trans. Am. Math. Soc. 163 (1972), 457-492.

34. A. Szankowski, $B(\mathcal{H})$ does not have the approximation property, Acta Math. 147 (1981), 89-108.

35. P. Wojtaszczyk, Banach spaces for analysts, Cambridge Studies in Advanced Mathematics, vol. 25 (Cambridge University Press, 1991). 